\title{
Computational electromagnetics for nanowire solar cells
}

\author{
Jan Kupec • Bernd Witzigmann
}

Published online: 24 February 2012

(C) Springer Science+Business Media LLC 2012

\begin{abstract}
This review article provides an overview of various novel nanowire array solar cells and highlights the aspects of electromagnetic simulations that are a valuable tool for understanding the optical processes leading to their distinct properties. As the computational methods commonly used for the task are well established, we focus on the question how numerical modeling can be used to assess the performance of a design and reveal the working principle of the devices. We conclude that scientific literature identifies numerical simulations as paramount for design and interpretation of experimental data.
\end{abstract}

Keywords Solar cell $\cdot$ Nanowire $\cdot$ Light trapping . Absorption enhancement - Emission - Detailed balance . Electromagnetics · Finite element · FDTD

\section{Introduction}

Photovoltaic devices with semiconductor nanostructures as the active material are under intense research for enabling more cost effective solar power generation as well as for powering future integrated photonic circuits. Many potential advantages were already identified [1]. These include intrinsic anti-reflective properties, large antenna apertures, high absorption at small material use compared to thin films, fabrication on low-cost substrates $[1,2]$ and multi-junction

J. Kupec ( $ه)$

Integrated Systems Laboratory, Gloriastrasse 35, 8092 Zurich, Switzerland

e-mail:kupec@iis.ee.ethz.ch

\section{B. Witzigmann}

Computational Electronics and Photonics, Wilhelmshöher Allee

71, 34121 Kassel, Germany bandgap engineering for efficient conversion of the incident light with reduced thermalization loss as strain relaxation increases design space [1].

Among numerous approaches for nanostructured absorbers including nano-gratings [3-5], nanoholes [6, 7], complex three-dimensional $[8,9]$ or even biomimetic designs [10-12], nanowires are especially in the focus.

In the past few year we have been observing the advent of many results on synthesis. Nanostructures are synthesized using both top-down approaches (e.g. reactive ion etching $[13,14]$ or chemical etching) or bottom-up approaches such as metalorganic vapor phase epitaxy $[15,16]$ or the vaporliquid-solid method [17, 18].

A variety of materials is being evaluated for their use in nanostructured photovoltaic devices, including group IV (silicon and germanium), III-V compound semiconductors (such as InP, GaAs, GaN, InAs, as well as their alloys), group II-VI (CdTe, CdS) semiconductor, dye-sensitized inorganic materials as well as organic semiconductors. An indepth review of silicon nanowire solar cells (including the fabricational aspects) was published in 2010 by Pen and Lee [19]. Compound nanowire solar cells are discussed in Ref. [20] and III-V nanowire growth in Refs. [21, 22].

For additional reviews on fabrication and experimental results of various nanowire architectures, the reader is referred to the papers by Lu [23], Tsakalakos [24], Hochbaum and Yang [25] and Fan et al. [26]. The most recent review papers, published in 2011, specifically on nanostructured solar cells include Garnett et al. [1], Dittrich et al. [27] and Musselman et al. [28].

Nanostructured devices can be grouped in different ways: From a fabricational point of view, the devices can be categorized by the deposition methods. Also the discussion of charge separation and carrier transport in III-V compound semiconductor nanowires is entirely different from carrier 

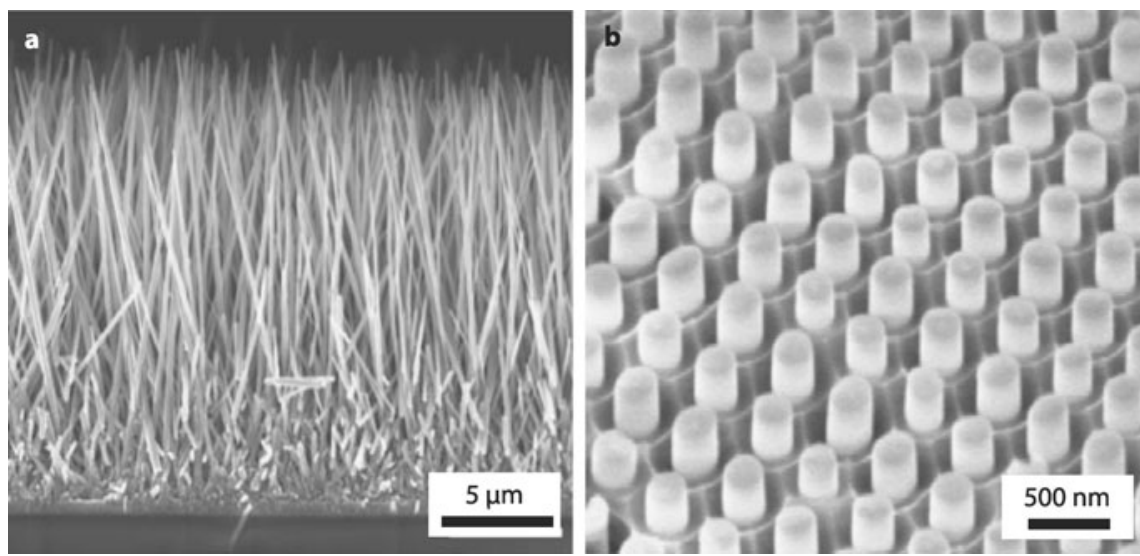

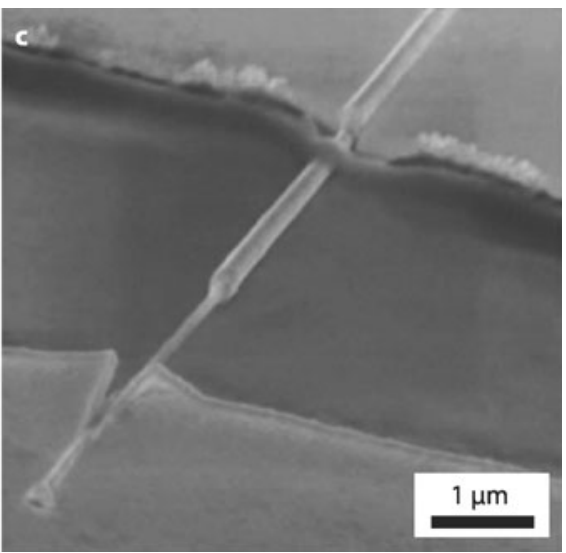

Fig. 1 SEM images of various types of nanowire solar cells. (a) randomly arranged nanowires with no long-range order, (b) nanopillar solar cell faeturing a long-range order triangular array, (c) single nanowire photovoltaic cell. Adapted by permission: (a) from Macmil-

transport in dye-sensitized or organic solar cells. From a computational electromagnetics perspective, however, we can classify the devices in three categories based on the geometry of the nanostructure, regardless of the material. For the purpose of illustration, we provide exemplary SEM micrographs of each category in Fig. 1. The categories are:

1. Devices based on randomly arranged nanowires exhibiting no order or only a limited near-range order. Randomly arranged silicon nanowires are presented in Refs. [32-37], gallium arsenide nanowires in Ref. [38], indium arsenide nanowires in Ref. [39], titanium dioxide dye-sensitized solar cells in Ref. [29] and zinc oxide dye-sanitized solar cells in Ref. [40].

2. Nanostructured devices showing long-range order. Literature reports a multitude of functioning devices or structures that could be used for photovoltaics after doping and contacting and thus constitute preliminary results. Examples include Si nanowire array solar cells built along the lines of Refs. [14, 41-43], silicon frustum nanorods [44, 45], dual-diameter germanium nanowires [46], CdTe/CdSe nanopillars [30], indium phosphide nanowires [15, 16, 47], indium phosphide/indium arsenide heterostructure nanowires [48] and many more, such as nanopillar-structured organic solar cells [49, 50]. Furthermore, there are structures that constitute hybrid approaches, such as a periodic square array with shorter randomly arranged wires in-between [51]. Compound semiconductor nanowires are also in the focus of research for light emitters [52-55], which have-as will be discussed in Sect. 4-contrary design goals to solar cells.

3. Single nanowire devices, which are intended to provide electric power in the nano-watt range for integrated photonics applications [56, 57]. Some notable approaches include core-shell silicon nanowires [58, 59], lian Publishers Ltd.: Nature, Ref. [29], copyright 2005, (b) from Macmillian Publishers Ltd.: Nature, Ref. [30], copyright 2009, (c) from Ref. [31], copyright American Institute of Physics, 2009

axial silicon nanowires $[60,61]$, core-shell group IIInitride nanowires [62], germanium nanowires [63] and GaAs single nanowire solar cells [31].

In this publication, we focus on rigorous computational methods yielding the spatially and spectrally or temporally resolved electric and/or magnetic field.

For all numerical methods providing a rigorous solution of the Maxwell equations the computational domain has to be limited in size as computational resources are finite. Although there are differences between the respective numerical methods, as of 2011, we can generally state that in practical terms the computational domain needs to be smaller than $100 \lambda^{3}$ in volume, where $\lambda$ is the shortest illumination freespace wavelength considered, to fit a mid-size commercially available compute server.

It is therefore necessary to either exploit (if applicable) periodicity and/or symmetry and/or the finite extensions of the structure to solve the Maxwell equations in a rigorous way. Devices of the first category are out of the scope of this paper. We want to note that those devices are successfully studied by statistical methods from particle scattering theory. For more information, the reader is referred to Refs. [64-66]. Furthermore, some authors introduce artificial periodicity with a unit cell containing from a few up to hundreds of nanowires, implicitly assuming that this measure does not introduce any spurious effect [34, 67]. In the following sections, we shall focus on devices either featuring long-range periodicity or small extensions in terms of wavelength.

Also, we want to point out that this publication only covers devices with nano-structures active regions and omits the discussion of thin-film or bulk solar cells with nanostructures merely employed as anti-reflective coatings [68-72]. 


\section{Methods}

In this section we summarize the simulation methods most commonly applied to nanostructured solar cells. In general, we differentiate between solving the source problem (SP) and the eigenvalue problem (EVP). For the SP an imposed illumination is given and the electromagnetic fields within the computational domain are determined. The SP directly yields absorptivity for a particular geometry, wavelength and angle of incidence.

In the EVP, oscillatory solutions of the Maxwell equations in the absence of an excitation are computed. The solutions of the EVP, the modes, are used in numerous publications to explain the behavior of nanostructured solar cells in various aspects (see Sects. 3 and 4). The calculation of waveguide modes for structures featuring translational invariance with respect to one direction, the calculation of discrete resonant states in a non-periodic 3D structure and the computation of the photonic band structure and states of 1D to $3 \mathrm{D}$ structures are all eigenvalue problems.

\subsection{Simulation methods}

One of the most common numerical methods for solving the Maxwell equations for nanostructured photovoltaic devices (and in fact any electromagnetic problem) is the FiniteDifference-Time-Domain (FDTD) method [73, 74]. In its original form, FDTD discretizes the spatial derivatives of differential operators in the Maxwell equations by expanding the field on a regular tensorial grid and integrates the temporal derivatives by time-stepping. The method is explicit, it does not require the solution to a large set of linear equations and can be parallelized [75] to be executed on high-performance low-cost graphical processing hardware [76, 77]. This fact constitutes a true advantage for the simulation of large devices. It a distinct disadvantage, however, that spectrally resolved complex refractive indices of the semiconductor materials as determined experimentally, cannot be directly incorporated into the simulation. As FDTD operates in the time domain, the impulse response of the dielectric material has to be obtained by $z$-transform $[78,79]$. Such methods often require analytical (e.g. Lorentzian) models which is a strong simplification of the dynamics and was observed to cause minor spurious effects in the simulation of nanowire array solar cells [80]. FDTD is applied to the problem by numerous authors $[9,42,46,80,81,83,84]$.

The Finite Element Method (FEM) can be formulated in both the time-domain (TD) as well as in the frequencydomain (FD). In the context of nanowire solar cell simulations it is most commonly used in the frequency domain (FD) formulation (see [85-87]). In contrast to FDTD, FEM is an approximation of the solution, not of the differential equation and can be applied to an unstructured mesh with locally varying refinement. The frequency-domain formulation naturally allows the use of frequency-dependent refractive indices. As a drawback, FEM requires the solution of a set of linear equations. On the other hand, FD-FEM allows for the computation of the eigenmodes of the system, naturally resolving even degenerate states, which are difficult to differentiate in a time-domain simulation with a Dirac-like excitation [88].

The Transfer Matrix Method (TMM) is a plane wave expansion of the electromagnetic fields relating the fields at the boundaries of a small sub-domain [89-92]. These methods are especially advantageous for structures containing large spaces of homogeneous media. The TMM is applied to the analysis of singe nanowire and nanowire array solar cells $[42,93]$ as well as other nanostructured designs including nanohole arrays [7].

An entirely different approach is pursued in the Rigorous Coupled Wave Analysis (RCWA) (more generally referred to as the Fourier-Modal method (FMM) [94]) where the fields are calculated in Fourier-space on the Fourierexpansion of the dielectric geometry [94-98]. Numerous authors make use of this method for their modeling [5, 36, 50, 99].

The further sophisticated Fast-Fourier-Factorization (FFF) ensuring faster convergence of the Fourier series of fields $[100,101]$ was also recently successfully applied to the analysis of absorption of nanowire array solar cells [102]. In its original formulation the FFF method was formulated for arbitrary periodic structures but can be reformulated for aperiodic geometries [100].

There is a variety of methods specifically designed for the efficient computation of photonic bandstructures, we refer to a review by Matias et al. [103]. Among the most popular, is the plane-wave expansion method implemented in the open-source package MIT Photonic Bands (MPB). Notably, Yip et al. employ this method to the design of dye-sensitized photonic crystal solar cell leveraging light field localization at the photonic bandedge [83, 84].

\subsection{Boundary conditions}

Depending on the devices under research, the numerical methods need to provide two classes of boundary conditions: (1) the simulation of nanostructured solar cells featuring a periodic geometry (of virtually infinite periodicity) requires periodic boundary conditions (PBC) reducing the computational domain to one unit cell, whereas (2) the simulation of single nanowire photovoltaic devices requires the simulation of open domains with the nanowire being embedded in a homogeneous medium or located on an infinite substrate. 


\subsubsection{Boundary conditions imposing periodicity}

For the former problem there exist PBCs where the solution corresponds to the structure obtained by translation of the computational domain (unit cell). Literature mentions such PBCs for FDTD [104-106] and FEM [107, 108] and also for FMM and TMM, where the relations between the fields can be readily incorporated.

However, for the simulation of many infinitely periodic structures, the implementation of PBCs is not necessary: In case the periodic geometry can be formed by mirroring a rectangular-shaped unit cell and only normally incident illumination is considered, the periodic boundary conditions at the sidewalls of the computational domain are trivially reduced to perfect electric conducting (PEC) and perfect magnetic conducting (PMC) surfaces. Such unit cells can be identified not only for gratings or rectangular-shaped nanowire arrays, but also in triangular or hexagonal arrays, making the trivial PEC and PMC boundary conditions sufficient to cover most periodic designs. Figure 2 illustrates the expansion of various array geometries by the PEC and PMC boundary conditions.

The applicability of PEC and PMC as the boundary conditions under the aforementioned conditions is not only favorable from an implementation point of view (PEC and PMC are trivial in both FDTD and FEM) but also allow for an important observation:

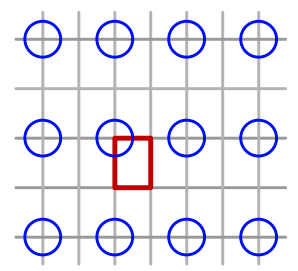

Rectangular

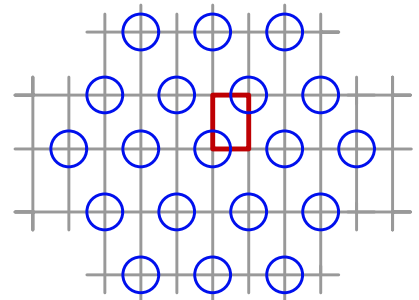

Triangular

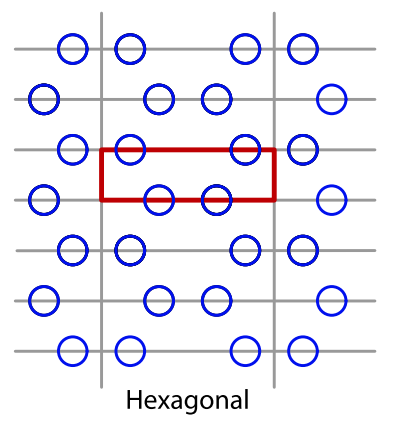

Fig. 2 (Color online) Computational domains ( $r e d$ ) for using PEC and PMC boundary conditions to expand infinitely extended array through mirroring at the domain sidewall for various array geometries. Grey lines mark surfaces in the array through which there is no flow of energy in case the PEC and PMC boundary conditions are applicable, i.e. normally incident light
On as PEC surface, with $\mathbf{n}$ being the normal vector, the following relations for the electric field $\mathbf{E}$ and magnetic field H hold:

$\mathbf{E} \times \mathbf{n}=0 \quad$ and $\quad \mathbf{H} \cdot \mathbf{n}=0$.

On the PMC surface the statement reads

$\mathbf{H} \times \mathbf{n}=0 \quad$ and $\quad \mathbf{E} \cdot \mathbf{n}=0$.

As the Poynting vector $\mathbf{S}=\mathbf{E} \times \mathbf{H}$ is orthogonal to both electric and magnetic field, we can conclude that $\mathbf{S} \cdot \mathbf{n}=0$ at both PEC and PMC boundaries. This fact is a strong statement with regard to the interpretation of simulation results: There is no flux of energy between neighboring unit cells (with the entire structure being expanded by mirroring the unit cell). Many nanostructured device are simulated with these boundary conditions or are simulated with PBC but fulfilling the symmetry of structure and excitation for the PEC and PMC being applicable. An example of the latter scenario is shown in Ref. [81] where PBCs are used while PEC and PMC boundary conditions would have been sufficient also allowing the reduction of the computational domain size by a factor of 4 as an additional benefit.

In case absorption enhancement is observed, in literature it is sometimes attributed to increased optical path lengths or the coupling to lateral photonic crystal modes [109]. Assuming that normally light is coupled to laterial guided photonic crystal modes at the $\Gamma$-point is formally correct, however, it is important to note that a guided photonic crystal mode at the $\Gamma$-point does not guide power along the structure.

The mode at the $\Gamma$-point rather constitutes a standing wave that is merely confined to the array. All power incident upon the unit cell is either absorbed, transmitted or reflected within the same unit cell with no communication in terms of energy exchange between neighbors. The same scenario occurs in photonic crystal membrane filters when illuminated at normal incidence [110].

\subsubsection{Open boundary conditions}

For the simulation of single nanowire solar cells, there exists a variety of boundary conditions for simulating open domains such as the Absorbing Boundary Condition (ABC) [111] or hybrid Finite Element/Boundary Element methods [112]. As simulations of nanostructured absorbers are focused on the absorption within the active material and not the far-field of the scattering from the structure, the use of Perfectly Matched Layers (PML) is possible [113]. A PML is not a boundary condition by itself, it is rather an artificial dielectric [114] with a gradual variation of its properties leading to high attenuation and low reflection. In case the PML is designed properly, the PML-cladded part of the computational domain contains only negligible field energy. Thus, the computational domain can be terminated with 
an arbitrary boundary condition. PML is applicable to the FDTD method as well as TD-FEM and FD-FEM or to any other method that can encompass a complex anisotropic dielectric. Most importantly, the concept of PML was also applied to the FMM [115] alleviating the periodicity implicitly imposed by the boundary condition in a similar manner as in FDTD and FEM. Therefore, Fourier-Modal methods are also applicable to single nanowire photovoltaic devices.

\subsection{Excitation}

The excitation of the system is either imposed by the boundary conditions or introduced by the flux of current along planes or Hertzian dipoles. The boundary conditions not only impose relations between the fields in the infinitely periodic structure, but also on the excitation. For frequencydomain methods, the structure is illuminated by coherent radiation. This fact may correspond to the illumination of solar cell by a laser, but it is in huge contrast to the actual properties of black-body radation such as originating from the sun. For those sources, the coherence length is approximately [116]

$l_{\mathrm{c}} \approx \frac{h c}{4 k_{\mathrm{B}} T}$

resulting in $l_{\mathrm{c}}=600 \mathrm{~nm}$ at $6000 \mathrm{~K}$. This aspect is implicitly compensated by the linearity of the system and the fact that solar cells are designed to have strong absorption, so the absorption occurs on a local scale.

\section{Absorption}

\subsection{Nanowire array devices}

Starting with the analysis of optical absorption in silicon nanowire array solar cells reported by $\mathrm{Hu}$ and Chen in 2007 [93], this type of devices attracted much attention. The publication focuses on nanometer diameters between $50 \mathrm{~nm}$ and $80 \mathrm{~nm}$ arranged in a square array of $100 \mathrm{~nm}$ center-tocenter distance illuminated by plane waves from normal and oblique directions [93]. To our best knowledge the authors are the first to report many unique properties of nanowire array solar cells including the intrinsic anti-reflective properties, small dependence of absorptivity on the angle of incidence for deviations from the normal axis and the fact, that sparse arrays can absorb virtually all of the incident light in some spectral ranges. For high energy photons (approx. $4 \mathrm{eV}$ ) the authors observed higher absorptance than in thin films, however, at photon energies close to the material bandgap (photon energy approx. $1.1 \mathrm{eV}$ ) the authors find, that the nanowire arrays within their design scope absorb less than the thin film [93]. As will be discussed later in this section, the latter conclusion arises form the nanowire diameter $\mathrm{Hu}$ and Chen investigated.

During the following years, similar results were published by various groups:

In 2009 Kelzenberg et al. used FDTD to study the absorptivity of Si microwires arranged in a square array finding that a sparse array of $14 \%$ geometric filling-factor results in the absorption of $66 \%$ of above-bandgap photons of the AM1.5g spectrum [81]. In 2010, Kelzenberg et al. applied the same method to the study of silicon nanowire array devices configured in different lateral arrangements [82].

In 2009 Lin and Povinelli reported that Si nanowires of $540 \mathrm{~nm}$ diameter arranged in a square array with lattice constant of $600 \mathrm{~nm}$ exhibit $72.4 \%$ more photogeneration current than a bare (without anti-reflective coating) thin film of equal thickness [42]. The high performance of the nanowires compared to a Si thin film is mainly caused by the array's intrinsic antireflective property (see Fig. 4a in Ref. [42]) and to a smaller extend by near-field absorption enhancement (see Fig. 4c in Ref. [42]). Because anti-reflective coatings are well understood and applied to all solar cells on industrial scale, the comparison with bare $\mathrm{Si}$ is biased, nonetheless, Lin and Povinelli showed the high potential nanostructures solar cells. In their publication [42] they also confirmed the absorptivity being virtually invariant of the polarization and direction of incidence up to angles of $\theta=30$ degrees (see Fig. 7 in Ref. [42]). A spherical cap spans a solid angle of $2 \pi(1-\cos (\theta))$. With the sun seen under an angular diameter of approx. 0.5 degrees, the device shown by Lin and Povinelli absorbs well for concentrations up to 3500 suns, which is well above the limits imposed by carrier transport.

For the nanowire array geometry, solving the source problem allowed the evaluation of various designs, however, the data did not reveal the optical processes leading to the particular spectral absorptivity. Instead, the discussion of the results was purely descriptive. Although the solution of the source problem demonstrated all properties such as intrinsic anti-reflective behavior and absorption enhancement, this situation was unsatisfactory, leading to a deeper investigation of the absorption process.

In 2008, Duche et al. attributed the absorption enhancement to slow Bloch modes, i.e. group velocity close to zero [109]. As noted in Sect. 2.2 for normal incident light as discussed in Ref. [109] there is no flow of energy along the array, at the $\Gamma$-point the bands are flat and $v_{\mathrm{g}}=\frac{\partial \omega}{\partial k}=0$. In their study, the authors identify a high density of Bloch states at the $\Gamma$-point for photon frequencies (energies) of interest as beneficial for absorption enhancement [109]. A minimal curvature $\alpha=\frac{\partial^{2} \omega}{\partial k^{2}}$ of the modal bands at $\Gamma$ is further highlighted [109]. Similar conclusions were reached by Lin and Povinelli [42].

In 2009, we (Kupec and Witzigmann) studied nanowire arrays illuminated with normally incident light. We showed 

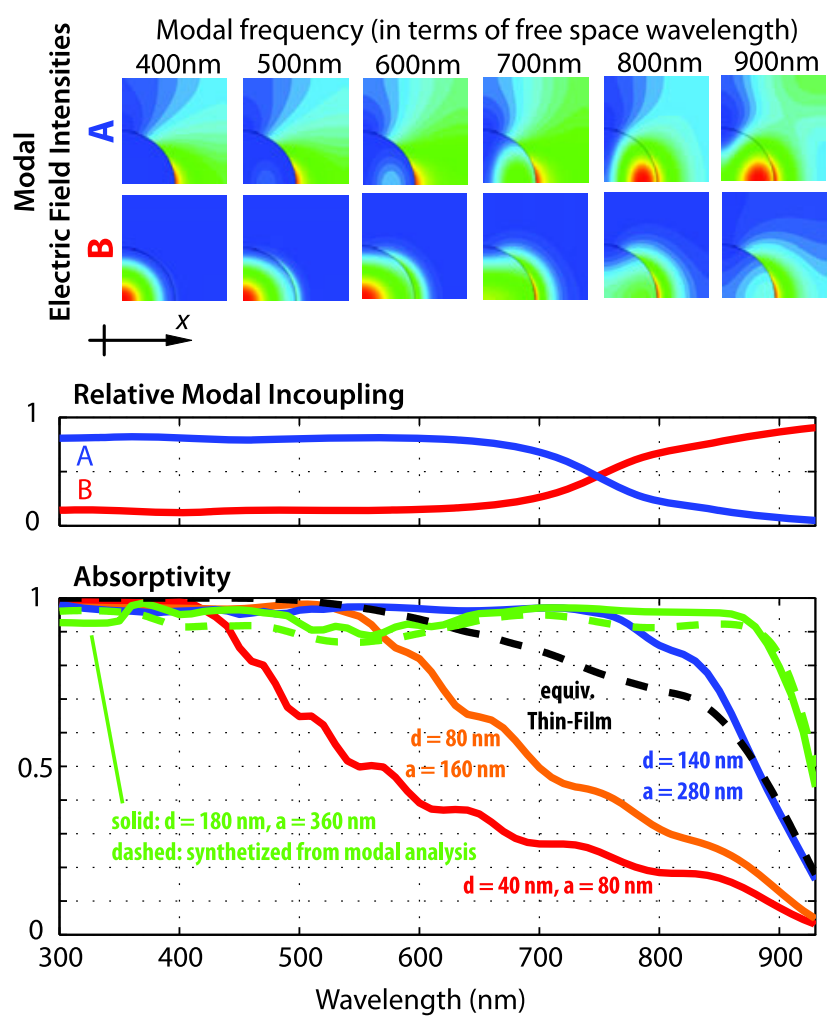

Fig. 3 (Color online) Modal and 3D FEM analysis of an InP nanowire array solar cell. Top: Modal field profiles at various frequencies expressed in terms of free-space wavelength and relative incoupling as defined in (8) in Ref. [87]. In a nutshell, the relative incoupling is a product of which one factor is the correlation between the modal field and the incident TEM plane wave and the other factor the transmission coefficient computed with the Fresnel equation for the transmission of light from free space of unity refractive index to a medium of modal effective refractive index. Bottom: spectral absorptivity of various array geometries with common nanowire height $2 \mu \mathrm{m}$ (colored solid lines). The geometric filling factor of all arrays is identical (0.196). Black dashed line: absorptivity of a perfectly AR-coated thin-film device of identical material use $(0.196 \cdot 2000 \mathrm{~nm})$, green dashed line: absorptivity computed with modal analysis $[86,87]$ using the dispersion data from mode A and B (see top). The data shown here is identical to Fig. 1 of Ref. [87]

that most of the light energy is travelling along the nanowires in discrete modes [86]. This observation is remarkable as the nanowires are only a few wavelengths long. In contrast to Refs. [42, 109] our method relates the dispersion relation (EVP) and absorptivity (SP) in a quantitative way. The method is described in Ref. [86], some exemplary results are shown in Fig. 3.

A comparison of the EVP modal analysis to 3D FD-FEM (SP) showed good agreement for the investigated geometries (compare solid green and dashed green curves in Fig. 3). We showed that nanowire arrays of too thin nanowires perform worse than a thin-film device (compare red and orange lines in Fig. 3). A too small nanowire diameter cannot be compensated by a dense packing (see Fig. 2 in Ref. [87]). Carefully engineering the dispersion relation can lead to significant absorption enhancement caused by wave-optical processes in which an increase of modal absoptivity compensates the decrease of material absorptivity for photon energies close to the bandgap [87].

Based on our modal analysis, our studies suggest that tapering the nanowires along their length can be used to implement two effects: First, building nanowires with thin top sections leads to better incoupling as the transition from free space plane wave, transverse electro-magnetic (TEM) light to waveguide modes is better impedance matched. Second, with regard to multi-junction nanowire solar cells, spectral splitting of absorptivity can be achieved in different sections of the structure.

Also, we have shown that for identical material use a carefully designed nanowire array solar cell can absorb more than thin-film device featuring an idealized perfect antireflective coating (see blue and green lines and black dashed line in Fig. 3) [87]. This statement is a stronger motivation for the nanowire architecture than in Ref. [93] or in Ref. [42] where mostly the anti-reflective properties of the nanowires contributed to their favorable performance.

Studying a similar geometry as discussed in Fig. 3, in 2010 a paper by Anttu and Xu [102] confirmed all major findings on nanowire array solar cells including the diameter being the limiting factor for long wavelength absorption, that cannot be compensated even with significantly larger material use. The authors also confirm the invariance of absorptivity with regard to angle and polarization of incidence for angles up to $\theta=55$ degrees [102], theoretically leading to uncompromised absorption for up to 11200 suns concentration. Paradoxically, as will be shown in Sect. 5, such a high angle of acceptance may even be impairing efficiency. Additionally, the authors propose a model relating the dispersion of the HE11 mode of a single lossy nanowire to the absorption decrease of an array at high wavelengths in a qualitative way [102].

In a recent publication [80], Diedenhofen et al. presented experimental results and FDTD simulations of an InP nanowire array. The nanowires feature a $2 \mu \mathrm{m}$ straight top of approx. $90 \mathrm{~nm}$ diameter and a tapered bottom of $1 \mu \mathrm{m}$ of height. The square array lattice constant is approx. $500 \mathrm{~nm}$. Measured angle-resolved specular reflectance of the structure confirms the intrinsic anti-reflective properties (see Fig. 4a in Ref. [80]). The structure under discussion [80] is similar to the structures investigated in Refs. [86, 87, 102]. The experimental data and simulations shown by Diedenhofen confirm both spectral splitting (compare Fig. 6 in Ref. [80] with Fig. 3) as well as low reflectance under oblique illumination (compare Fig. 4a in Ref. [80] with Fig. 2 in Ref. [102]).

In order to further improve the anti-reflective properties of nanowire arrays, nanowire tapering for better impedance matching was performed experimentally by Fan et al. [46]. 
a
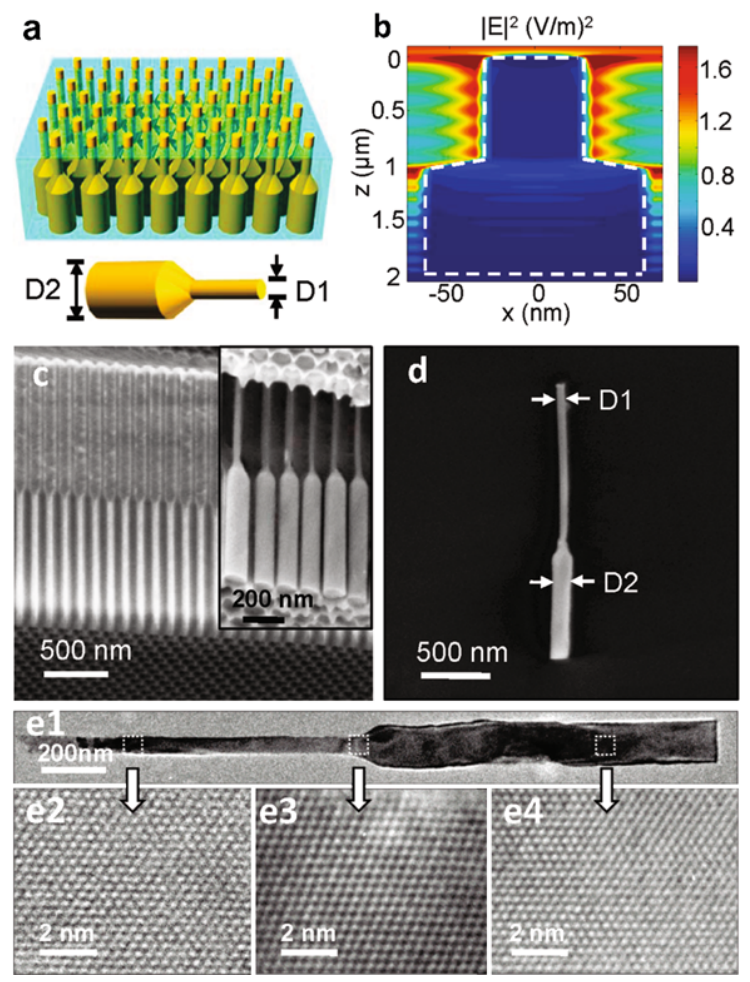

Fig. 4 Ge dual-diameter nanowire array. (a) schematic view, (b) simulated cross-sectional electric field intensity at $800 \mathrm{~nm}$ wavelength, (c, d) cross-sectional SEM images, (e1-e4) transmission electron microscopy images showing the single crystal structure at various positions. Reprinted with permission from Ref. [46]. Copyright American Chemical Society, 2010

They demonstrated the fabrication and numerical simulation of dual-diameter germanium nanowires. Their device is shown in Fig. 4. The authors fabricated single and dual diameter nanowire arrays and measured their respective absorptivity. The measurement data is shown in Fig. 5. The desired effect was achieved, the thin wires in the top pre-direct the light into the waveguide modes, the bottom large diameter section yields good absorption. Note that the bandgap energy of $\mathrm{Ge}$ is $0.67 \mathrm{eV}$, the absorptivity droop of the $60 \mathrm{~nm}$ and $80 \mathrm{~nm}$ geometry is entirely caused by wave optical phenomena alone (see Fig. 5 also compare with Fig. 3).

While many computational studies focus on nanowire arrays, a paper on the inverse structure-nanohole arrayswas published by Han and Chen [7] in 2010. The authors showed that for identical material use both nanowire and nanohole arrays exceed the absorptivity of bulk and that for certain geometries, nanoholes can outperform the nanowire architecture [7]. The authors claim that a nanohole array cSi design requires only $8.3 \%$ of material to achieve the photogeneration of a $0.3 \mathrm{~mm}$ c-Si solar cell under AM1.5 illumination. Independently and around the same time, Peng et al. presented a functioning c-Si nanohole array solar cell with a conversion efficiency of $9.51 \%$ under 1 sun AM1.5g light [6].

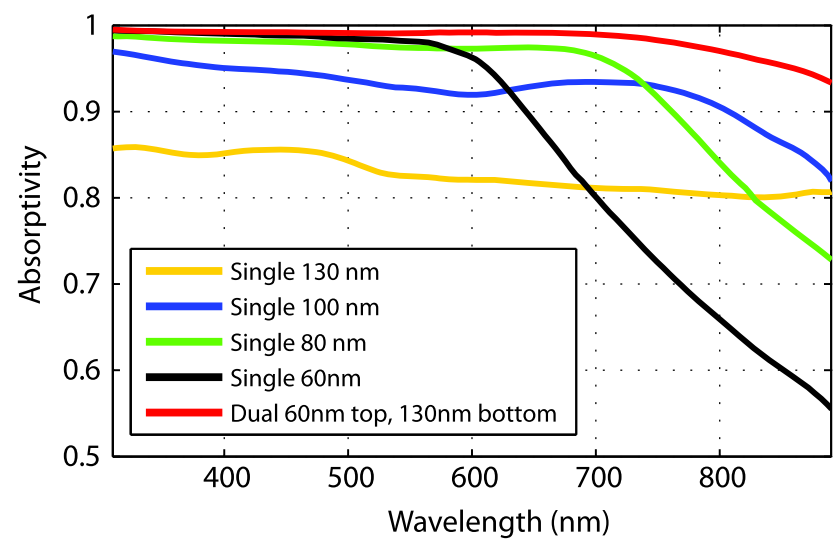

Fig. 5 Experimental absorptivity spectra of germanium nanowires for various diameters with constant unit cell size. The thin nanowires offer good incoupling but exhibit low absorptivity at long wavelengths, the dense array exhibits high reflectivity (also see Fig. 2a in the original publication [46]). The dual diameter design unifies the low reflectivity of the $60 \mathrm{~nm}$ geometry with the excellent absorptivity of the $130 \mathrm{~nm}$ device. Data obtained with permission from Ref. [46]. Copyright American Chemical Society, 2010

As observed commonly in literature, a nanowire array solar cell can absorb more light than incident on the projected surface of the nanowire. This result is important as it legitimates the nanowire architecture as potentially providing efficiencies exceeding current planar devices, but also it highlights some caveats in the interpretation of experimental data: As determined by simulation the InP nanowire solar cell designed by Goto et al. [47] absorbs most of the abovebandgap photons [117]. This fact contributes to the high efficiency of $3.4 \%$ observed in measurements, however, due to the near-field absorption enhancement, the authors' projection of the efficiency to $12.3 \%$ by scaling with the inverse geometric filling factor [47] is not applicable.

\subsection{Single nanowire photovoltaic devices}

For integrated photonic applications singe nanowire photovoltaic devices are often envisioned to be illuminated from the side. The light can be submitted to the nanowire either by a waveguide or, in a more basic configuration, can travel though a homogeneous medium such as free space. The wave-optical properties of the latter setup were extensively examined by Cao et al. [63]. They observed in experiment as well as numerical modeling that the absorption spectra of germanium nanowires illuminated from the side with plane waves exhibit features that cannot be related to the dispersion properties of the bulk material [63]. Most notably the authors found that the nanowires can capture light from a larger area than their physical projected cross-section (diameter multiplied by length). The authors refer to the ratio between absorption cross-section and the physical cross-section as absorption efficiency. Borrowing 


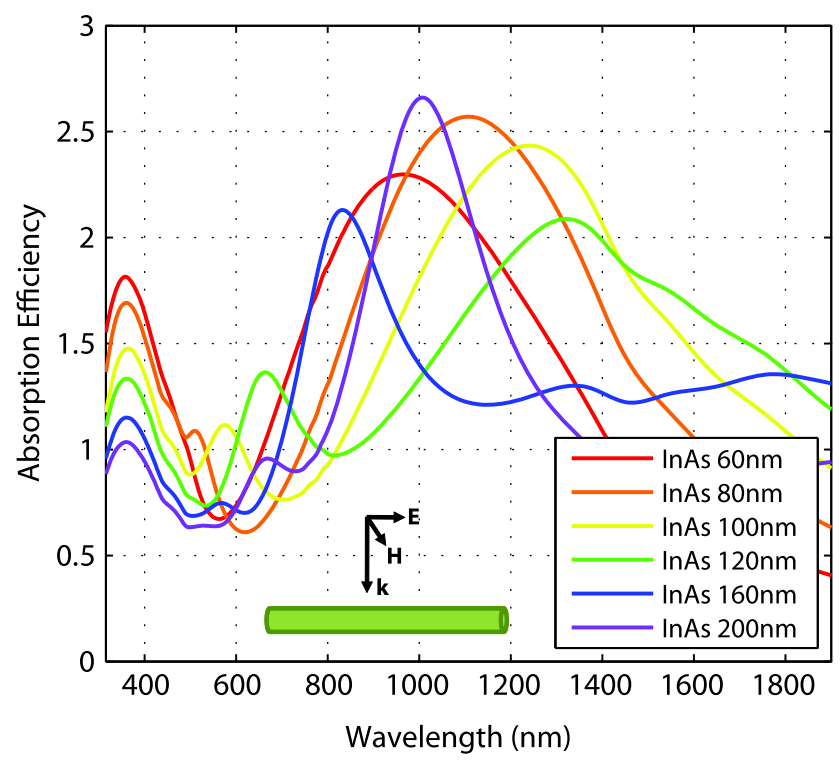

Fig. 6 Absorption efficiency as defined in Ref. [63] for sideways TM-illuminated InAs nanowires in free space. Illumination by plane wave schematically illustrated in the inset. Depending on the illumination wavelength, the nanowire aperture varies in size by a factor of 4 . The aperture of 1 is defined as the radiation into free space through the area of the projected nanowire

terms from antenna design, alternative nomenclatures are antenna/nanowire aperture/cross-section [118]. The authors report that the absorption efficiency is highly wavelength and incidence angle/polarization dependent, both, exceeding unity as well as approaching zero.

Cao et al. relate the results of the numerical simulation to analytical calculations of the transverse electric (TE) and transverse magnetic (TM) dielectric fiber modes. Depending on the polarization of the illumination under normal incidence, the respective modes at cut-off are excited in nanowire. As the modes are not perfectly confined to the nanowire but their field energies extend to the surrounding, incoupling of optical power into the modes occurs and gives rise to leaky mode resonance (LMR)-induced absorption enhancement [63]. Good agreement between the modal resonance frequencies and large absorption efficiencies was observed.

Although the experimental work and the simulations by Cao et al. were performed for Ge nanowires, their qualitative conclusions are valid for any nanowires of similar dimensions. Using FD-FEM we reproduced the numerical computations from [63] for single InAs nanowires of various diameters illuminated from the side. The results are plotted in Fig. 6. Our simulations confirm the conclusions claimed by Cao et al.

For the purpose of providing better insight, Fig. 7 shows the streamline trajectories along the real part of the Poynting vector $\frac{1}{2} \Re\left\{\mathbf{E} \times \mathbf{H}^{*}\right\}$ for notably large and small absorption

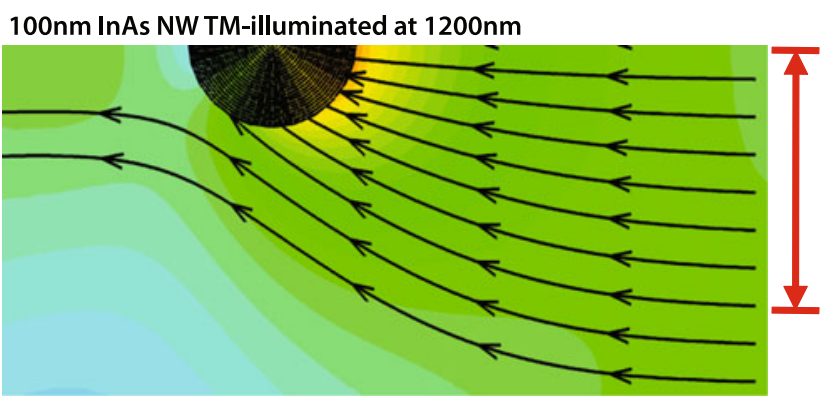

100nm InAs NW TM-illuminated at 700nm

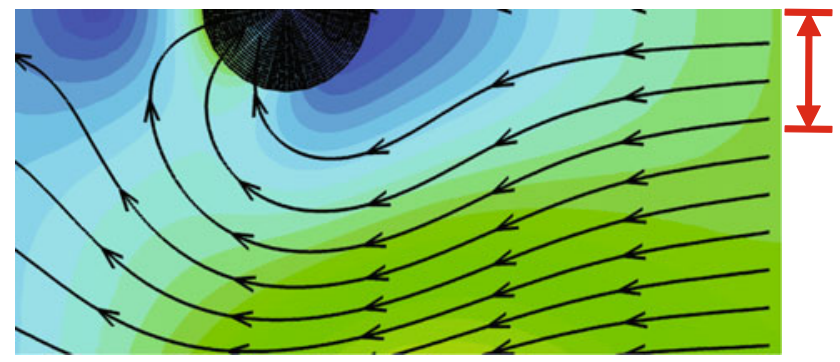

Fig. 7 Streamlines of trajectories of the real-power Poynting vector fit a $100 \mathrm{~nm}$ diameter InAs nanowire illuminated normally from the side with TM-polarized light. Refer to Fig. 6 for quantitative results. The approximative size of the effective nanowire cross-section/aperture is highlighted. Note that for $700 \mathrm{~nm}$ there is reflection compared to radiation to free space, that is why the streamlines from an are larger than the projected nanowire side-surface are captured while the quantitative data in Fig. 6 suggests the contrary. Illustration serves qualitative statement, no absolute values of colorbar are provided. Refer to Fig. 6 for quantitative data. Only a fraction of the computational domain is shown explaining reentrant streamlines in the illustration

efficiency occurring in the same structure at various wavelengths.

These near field wave-optical phenomena pose a serious difficulty for the evaluation of experimental results. There are numerous publications presenting experimental data and efficiency figures for single nanowire photovoltaic devices [31, 119]. Dong et al. report a maximum efficiency of approx. $0.19 \%$ in their $\operatorname{In}_{x} \mathrm{Ga}_{1-x} \mathrm{~N}$ core-shell nanowire solar cell [119]. Although the power density of the illumination source and the IV-curve are known, it is difficult to assess the impact of various mechanisms of efficiency decrease acting on absorption, carrier generation, carrier separation and contacting, because the effective nanowire aperture is unknown.

Borgström et al. report axial InP nanowire pn-junctions for the use in a multi-junction nanowire solar cell [120]. After the growth process, a single nanowire was aligned horizontally to a silicon substrate (see Fig. 9b in Ref. [120]) [120, 121]. The authors present IV-characteristics for various excitation power densities originating from a $532 \mathrm{~nm}$ Nd:YAG laser (Fig. 9b in Ref. [120]). By assuming that the current is generated in the depletion region of the nanowire the authors conclude an efficiency of about $6 \%$. Numerical simulations of the near-field absorption as well as carrier 
transport could help to verify the assumptions made in their analysis.

Similarly, it would be beneficial to quantify the contribution of LMR absorption enhancement to the efficiency of $4.5 \%$ reported by Colombo et al. [31] in a GaAs single nanowire device.

\section{Emission-a current challenge}

Apart from experimental aspects of fabricating nanostructured solar cells economically, reliably, with sufficient surface quality, doping levels and many other important parameters, the optical modeling of nanostructured solar cells still poses many challenges as an enabler for successful large scale deployment. Although the absorptivity of a nanostructured device is an important benchmark, ultimately the efficiency is the important figure of merit.

In order to asses the efficiency, the absorptivity data is submitted as an input parameter to simulations of electronic transport. Literature reports a variety of electronic simulations ranging from semi-analytical models such as detailed balance [122, 123], circuit-level simulations [124] up to 3D drift-diffusion modeling [5, 81, 125]. Although these models vary in their respective degree of sophistication, all these models need to include the fact that under steady-state operation, carrier generation and carrier extraction plus recombination are in balance $[122,126]$.

Among many, one pathway is radiative recombination. In a real bulk or thin-film solar cell, radiative recombination is of minor importance compared to non-radiative processes in bulk or on surfaces.

Non-radiative surface recombination is particularly critical for the performance of nanowire solar cells due to their high surface-to-volume ratio and must not be ignored in modeling. A good introduction is provided in Ref. [127]. Because its treatment does not involve computational electromagnetics, its discussion is beyond the scope of this paper.

In contrast to all other recombination pathways, radiative recombination is not merely a parasitic effect, it is rooted in semiconductor thermodynamics and is the limiting recombination mechanism in an ideal solar cell $[122,126]$.

The spatially resolved rate of radiative recombination is proportional to the local density of photon states (LDOS). Radiative recombination is of minor importance in planar solar cells due to the dominance of the other pathways. Regarding nanostructured solar cells, such a statement cannot be made a-priori as nanostructures are known to exhibit LDOS enhancement. This effect is commonly called Purcell effect $[128,129]$. In fact, nanostructures are under intense research dedicated to improving the efficiency of light emitting diodes (LEDs), which benefit from an enhancement of radiative recombination compared to the non-radiative pathways. But contrary to LEDs, the efficiency of a solar cell decreases in case any recombination pathway rate increases.

Most notably, very similar structures (see Fig. 8) are investigated for nanostructured LEDs and for nanostructured solar cells by different parts of the scientific community, both claiming beneficial properties for the respective application. As an intriguing example the III-V heterostructure nanopillar LED reported by Chuang et al. [53] bears strong similarity to a Si nanocone array photovoltaic device shown by Lu and Lal [45]. Although both devices consist of different materials and have deviating dimensions, none of the publication contains a computational optimization of the geometry with respect to the LDOS, therefore it is unclear whether the geometries are optimal for their respective purpose as both publications are implicitly claiming conflicting advantages of this type of geometry. As shown experimentally, nanowires can reduce photoluminescence decay time and thus enhance radiative recombination [130]. Similarly, nanohole arrays are under research for solar cells as well as for LEDs for their improved light extraction [131-135]. For a concisive review on emission enhancement in nanostructured LEDs we refer to Wiesmann et al. [136].

Focusing on nanowire solar cells, while Kuo et al. identified the HE11 mode of a single nanowire to be responsible for ultraefficient outcoupling of light from a single nanowire LED [55], Anttu identified the very same mode to be responsible for absorption enhancement in the nanowire solar cell [102].

The LDOS can be computed either via the dyadic Green's function [137] or through a complete modal expansion [129, 138]. The computation via the dyadic Green's function requires to probe the nanostructure with Hertzian dipoles, solving the SP for every position. Both approaches require sophisticated methods: To compute the LDOS in a nanostructured array via the dyadic Green's function, one needs to keep in mind that PBCs, do not only expand the geometry of the unit cell but also the sources within. The computation via the eigenmodes of the structure bears the uncertainty of truncating the series of modes. The calculation of LDOS enhancement has already received much attention and is still under intense research [138-142].

As a consequence of the reciprocity principle in electromagnetics [118] in general and reciprocity between photovoltaic quantum efficiency and electroluminescent emission of solar cells $[143,144]$ in particular, a complete study of the device also needs to include the emission enhancement of the radiative recombination caused by the photonic properties of the nanostructures. It is a common observation that the effective nanowire aperture exceeds its physical footprint and therefore it is necessary to assume that the process of emission also experiences enhancement at least in the direction from which light was incident in the absorption calculation. In Ref. [87] we highlighted that for geometric optical 


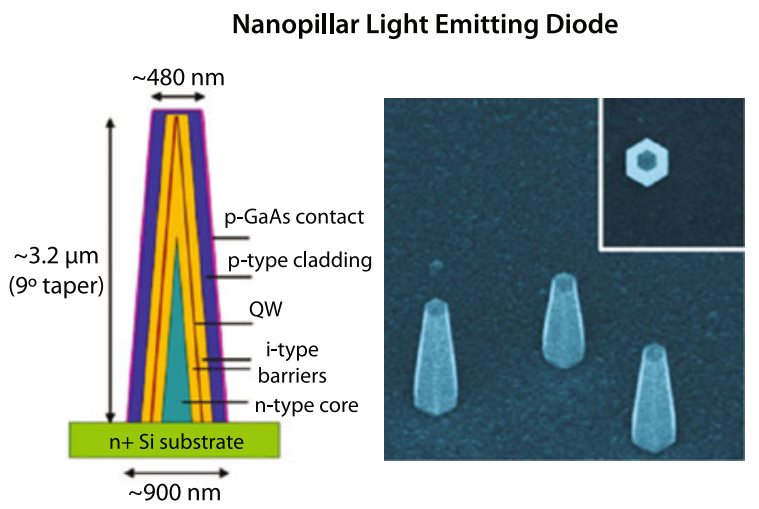

Fig. 8 Left: schematic drawing, dimensioning and material composition of a conical nanopillar LED and the micrograph of the fabricated device (adapted with permission from Ref. [53]), right: schematic drawing of a conical nanopillar Si solar cell (adapted with permission

concentrators such as a Fresnel lens the discussion of emission enhancement is trivial [87, 122, 123, 145-147]. For nanostructured devices, however, the ray-optical approach formulated in those references is not applicable. The necessity of wave-optical treatment applies to all nanostructured devices featuring absorption enhancement induced by nearfield concentration of any type $[3,42,63,86,87,102,148-$ 150].

In 2010, we reported a semi-analytical detailed balance efficiency calculation with the inclusion of LDOS enhancement. We computed the latter using 3D FD-FEM by simulating a finite section of the nanowire array [87]. The effects of LDOS enhancement in the nanostructure can be readily incorporated in 3D drift-diffusion simulators [151, 152], however, to our best knowledge, as of April 2011 there are no publications reporting this. Therefore, an efficiency analysis of nanostructures solar cells including both absorption and emission enhancement remains for further research.

\section{Light trapping}

In the body of scientific literature, the term 'light trapping' is used in two slightly different connotations: The first use alludes to the absorption of light. In this context light trapping means the increase of the effective optical thickness of the absorber material. For this definition, light trapping shall occur for the entire spectrum of incident light in which absorption occurs. The term is used in this meaning in numerous publications:

Mallick et al. propose a nanocavity solar cell [99], Ferry et al. employ plasmonic scatterers to increase absorption [153], Hu and Chen refer to light trapping as a measure that can improve absorptivity of their array designs [93].

On the other hand, particularly in literature on thin-film and concentrator solar cells, the term light trapping refers
Nanopillar Solar Cell
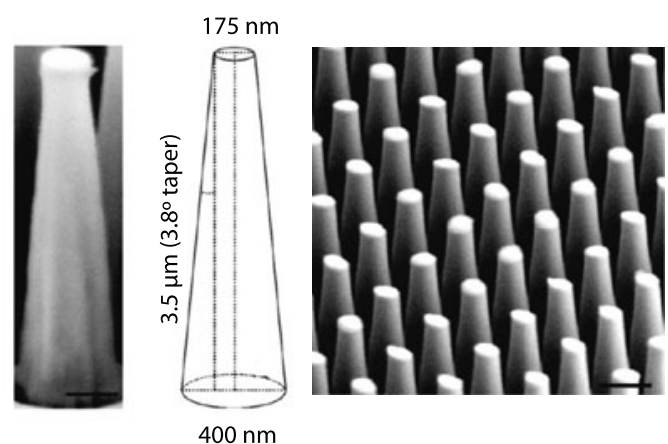

from Ref. [45]). The geometry of both devices is similar although the design goals for their respective application are contradicting. Copyright, American Chemical Society, 2010

to measures for the reduction of the LDOS within the active region of the device. In a ray-optical picture this means to restrict the solid angle into which radiative recombination can emit photons [154]. In this sense, light trapping is mostly required in the spectral regime in which emission occurs, i.e. at bandgap energy and a few $k_{\mathrm{B}} T$ above. This definition is used by various authors in the context of directionally selective filters [155-158].

In both definitions light trapping is beneficial for efficiency and it is common to both contexts that trapping of near-bandgap photons is desired regardless whether they arise from non-absorbed, scattered incident light or radiative recombination. By virtue of the reciprocity principle, both definitions are interweaved, however, there remains a subtle difference because each definition also can motivate different measures: While the former definition requires to particularly enhance absorption for photon energies near the bandgap at all cost, the latter definition leads to considerations of deliberately sacrificing near bandgap absorption to quench radiative recombination.

The idea of restricting the angle of emission to increase radiative lifetime can be tracked back to the initial paper of Shockley and Queisser [122]. In fact the long practiced measure of placing a metallic mirror at the back of the solar cell already constitutes light trapping along the latter definition.

It is, however, an ongoing challenge to achieve a narrow confinement. Peters et al. reported in 2011 experimental results on a germanium bulk solar cell with a directionally selective filter, they observed an increase of carrier generation of approx. 33\% near the bandgap of Ge [158]. Nonetheless the authors reported a negative result with respect to their objective as they could not detect a trapping of photons generated by radiative recombination and no suppression of the radiative process was achieved [158]. The beneficial effect was caused by trapping of scattered incident light, i.e. the 
directionally selective filter resulted in light trapping in the former sense instead of the latter [158].

Some recent studies on the topic can be found in Refs. [159-161] also discussing the potential photonic crystal structures for implementing light trapping according to the second definition. In 2008, Ulbrich et al. identified two main requirements for such a photonic structure [161]. Applied to photovoltaic devices with a nanostructured absorber, the requirements read: The structure must permit strong absorption from a narrow solid angle from which sunlight is incident and in all other direction the structure must provide a photonic stop band to suppress the escape non-absorbed scattered light as well as to quench radiative recombination. Ideally, this photonic stop band covers the entire spectrum of incident light and the entire $4 \pi$ unit sphere except for the small acceptance solid angle for the incident illumination.

For the engineering of light trapping, nanostructures can have an additional benefit: Recently it was shown that quantum wells in nanostructured solar cells can restrict the possible dipole orientation along which radiative recombination occurs $[162,163]$. The design of the proper structure constitutes a challenge for computational electromagnetics used for the simulation of nanostructured solar cells, opening a broad field of future research endeavors [164, 165].

\section{Conclusion}

In this paper, the electromagnetic modeling of nanostructured photovoltaic devices has been discussed. We related the results of various computational studies and showed how the design as well as the interpretation of experimental data can benefit from electromagnetic simulations. There is consensus in the body of scientific literature, that the distinct wave-optical features of nanostructured solar cells require a rigorous solution of the Maxwell equations and that calculations based on geometrical assumptions alone cannot encompass the discussed phenomena. Although the absorption behavior of nanostructured solar cells can be readily modelled and is well understood, the emission behavior and the design of optimized light trapping largely remains to be investigated. Only the inclusion of emission and absorption effects with the same level of sophistication of their modeling will allow a concise analysis of the various geometries.

Acknowledgements The research outlined in this publication was conducted within the AMON-RA project (further information can be found online at http://www.amonra.eu), grant agreement FP7-214814-1.

\section{References}

1. McGehee, M., Brongersma, M., Cui, Y., Garnett, E.: Ann. Rev. Mater. Res. 41(1), 269 (2011)
2. Logeeswaran, V., Katzenmeyer, A., Islam, M.: IEEE Electron Devices 57(8), 1856 (2010)

3. Cao, L., Fan, P., Vasudev, A., White, J., Yu, Z., Cai, W., Schuller, J., Fan, S., Brongersma, M.: Nano Lett. 10(2), 439 (2010)

4. Wang, W., Wu, S., Reinhardt, K., Lu, Y., Chen, S.: Nano Lett. 10(6), 2012 (2010)

5. Peters, M., Rüdiger, M., Bläsi, B., Platzer, W.: Opt. Express 18(104), A584 (2010)

6. Peng, K.Q., Wang, X., Li, L., Wu, X.L., Lee, S.T.: J. Am. Chem. Soc. 132(20), 6872 (2010)

7. Han, S., Chen, G.: Nano Lett. 10(3), 1012 (2010)

8. Myers, B., Bernardi, M., Grossman, J.: Appl. Phys. Lett. 96, $071902(2010)$

9. Chen, J., Li, E., Chen, L.: Prog. Electromagn. Res. 17, 1 (2011)

10. Zhang, W., Fan, T., Gu, J., Ding, J., Wang, H., Guo, Q., Ogawa, H.: Chem. Mater. 21(1), 33 (2009)

11. Parker, A., Townley, H.: Nat. Nanotechnol. 2(6), 347 (2007)

12. Vukusic, P., Sambles, J.: Nature 424(6950), 852 (2003)

13. Wang, H., Sun, M., Ding, K., Hill, M.T., Ning, C.Z.: Nano Lett.

14. Seo, K., Wober, M., Steinvurzel, P., Schonbrun, E., Dan, Y., Ellenbogen, T., Crozier, K.: Nano Lett. 11(4), 1851 (2011)

15. Heurlin, M., Wickert, P., Fält, S., Borgström, M.T., Deppert, K., Samuelson, L., Magnusson, M.H.: Nano Lett. 11(5), 2028 (2011)

16. Kitauchi, Y., Kobayashi, Y., Tomioka, K., Hara, S., Hiruma, K., Fukui, T., Motohisa, J.: Nano Lett. 10(5), 1699 (2010)

17. Kim, D., Lee, C., Zheng, X.: Nano Lett. 10(3), 1050 (2010)

18. Wu, Y., Yang, P.: J. Am. Chem. Soc. 123(13), 3165 (2001)

19. Peng, K., Lee, S.: Adv. Mater. (2010)

20. Sun, K., Kargar, A., Park, N., Madsen, K.N., Naughton, P.W., Bright, T., Jing, Y., Wang, D.: IEEE J. Sel. Top. Quantum Electron. 17, 1033 (2011)

21. Gao, Q., Tan, H.H., Jackson, H.E., Smith, L.M., Yarrison-Rice, J.M., Zou, J., Jagadish, C.: Semicond. Sci. Technol. 26(1), 014035 (2011)

22. Tomioka, K., Tanaka, T., Hara, S., Hiruma, K., Fukui, T.: IEEE J. Sel. Top. Quantum Electron. 99, 1 (2010)

23. Lu, W., Lieber, C.: J. Phys. D 39, R387 (2006)

24. Tsakalakos, L.: Mater. Sci. Eng. Rev. 62(6), 175 (2008)

25. Hochbaum, A., Yang, P.: Chem. Rev. 110(1), 527 (2009)

26. Fan, Z., Ruebusch, D., Rathore, A., Kapadia, R., Ergen, O., Leu, P., Javey, A.: Nano Res. 2(11), 829 (2009)

27. Dittrich, T., Belaidi, A., Ennaoui, A.: Sol. Energy Mater. Sol. Cells 95(6), 1527 (2011)

28. Musselman, K., Schmidt-Mende, L.: Green 1(1), 7 (2011)

29. Law, M., Greene, L., Johnson, J., Saykally, R., Yang, P.: Nat. Mater. 4(6), 455 (2005)

30. Fan, Z., Razavi, H., Do, J., Moriwaki, A., Ergen, O., Chueh, Y., Leu, P., Ho, J., Takahashi, T., Reichertz, L., et al.: Nat. Mater. 8(8), 648 (2009)

31. Colombo, C., Heiß, M., Grätzel, M., i Morral, A.: Appl. Phys. Lett. 94, 173108 (2009)

32. Tsakalakos, L., Balch, J., Fronheiser, J., Korevaar, B., Sulima, O., Rand, J.: Appl. Phys. Lett. 91, 233117 (2007)

33. Tsakalakos, L., Balch, J., Fronheiser, J., Shih, M., LeBoeuf, S., Pietrzykowski, M., Codella, P., Sulima, O., Rand, J., Kumar, A., et al.: Photovolt. Energy Convers. 1, 111 (2006)

34. Tsakalakos, L., Balch, J., Fronheiser, J., Shih, M., LeBoeuf, S., Pietrzykowski, M., Codella, P., Korevaar, B., Sulima, O., Rand, J., et al.: J. Nanophotonics 1, 13552 (2007)

35. Chen, C., Jia, R., Yue, H., Li, H., Liu, X., Ye, T., Kasai, S., Tamotsu, H., Wu, N., Wang, S., et al.: J. Vac. Sci. Technol. B 29(2), 021014 (2011)

36. Tham, D., Heath, J.: Nano Lett. 10(11), 4429 (2010)

37. Gunawan, O., Guha, S.: Sol. Energy Mater. Sol. Cells 93(8), 1388 (2009) 
38. Czaban, J., Thompson, D., LaPierre, R.: Nano Lett. 9(1), 148 (2008)

39. Wei, W., Bao, X., Soci, C., Ding, Y., Wang, Z., Wang, D.: Nano Lett. 9(8), 2926 (2009)

40. Xu, C., Wang, X., Wang, Z.: J. Am. Chem. Soc. 131(16), 5866 (2009)

41. Garnett, E., Yang, P.: Nano Lett. 10(3), 1082 (2010)

42. Lin, C., Povinelli, M.: Opt. Express 17(22), 19371 (2009)

43. Gunawan, O., Wang, K., Fallahazad, B., Zhang, Y., Tutuc, E., Guha, S.: Progr. Photovoltaics Res. Appl. 19(3), 307 (2011)

44. Tsai, M., Tseng, P., Chen, H., Kuo, H., Yu, P.: Opt. Express 19(101), A28 (2011)

45. Lu, Y., Lal, A.: Nano Lett. 10(11), 4651 (2010)

46. Fan, Z., Kapadia, R., Leu, P.W., Zhang, X., Chueh, Y.L., Takei, K., Yu, K., Jamshidi, A., Rathore, A.A., Ruebusch, D.J., Wu, M., Javey, A.: Nano Lett. 10(10), 3823 (2010)

47. Goto, H., Nosaki, K., Tomioka, K., Hara, S., Hiruma, K., Motohisa, J., Fukui, T.: Appl. Phys. Express 2(3), 5004 (2009)

48. Mohan, P., Motohisa, J., Fukui, T.: Appl. Phys. Lett. 88, 133105 (2006)

49. Ko, D., Tumbleston, J., Zhang, L., Williams, S., DeSimone, J., Lopez, R., Samulski, E.: Nano Lett. 9(7), 2742 (2009)

50. Duche, D., Drouard, E., Simon, J., Escoubas, L., Torchio, P., Le Rouzo, J., Vedraine, S.: Sol. Energy Mater. Sol. Cells 95, 518 (2011)

51. Park, K., Guo, Z., Um, H., Jung, J., Yang, J., Lim, S., Kim, Y., Lee, J.: Opt. Express 19(101), A41 (2011)

52. Tomioka, K., Motohisa, J., Hara, S., Hiruma, K., Fukui, T.: Nano Lett. 10(5), 1639 (2010)

53. Chuang, L., Sedgwick, F., Chen, R., Ko, W., Moewe, M., Ng, K., Tran, T., Chang-Hasnain, C.: Nano Lett. 11(2), 385 (2011)

54. Chuang, L., Chen, R., Sedgwick, F., Ko, W., Ng, K., Tran, T., Chang-Hasnain, C.: Proc. Opt. Soc. Am. (2010). http://www. opticsinfobase.org/abstract.cfm?URI=CLEO-2010-CMFF6

55. Kuo, M., Kim, Y., Hsieh, M., Lin, S.: Nano Lett. 11(2), 476 (2011)

56. Wehrspohn, R.: ChemSusChem 1(3), 173 (2008)

57. Tian, B., Kempa, T., Lieber, C.: Chem. Soc. Rev. 38(1), 16 (2009)

58. Tian, B., Zheng, X., Kempa, T., Fang, Y., Yu, N., Yu, G., Huang, J., Lieber, C.: Nature 449(7164), 885 (2007)

59. Liu, W., Oh, J., Shen, W.: IEEE Electron Device Lett. 32(1), 45 (2011)

60. Kempa, T., Tian, B., Kim, D., Hu, J., Zheng, X., Lieber, C.: Nano Lett. 8(10), 3456 (2008)

61. Kelzenberg, M., Turner-Evans, D., Kayes, B., Michael, A., Putnam, M., Lewis, N., Atwater, H.: Nano Lett. 8(2), 710 (2008)

62. Dong, Y., Tian, B., Kempa, T., Lieber, C.: Nano Lett. 9(5), 2183 (2009)

63. Cao, L., White, J.S., Park, J.S., Schuller, J.A., Clemens, B.M., Brongersma, M.L.: Nat. Mater. 8(8), 643 (2009)

64. Mehta, A.: Granular Matter: An Interdisciplinary Approach. Springer, Berlin (1994)

65. Ferber, J., Luther, J.: Sol. Energy Mater. Sol. Cells 54(1-4), 265 (1998)

66. Rothenberger, G., Comte, P., Gratzel, M.: Sol. Energy Mater. Sol. Cells 58(3), 321 (1999)

67. Altermatt, P., Yang, Y., Langer, T., Schenk, A., Brendel, R.: In: Proc. Photovoltaic Specialists Conference, pp. 972-977 (2009)

68. Xi, J., Kim, J., Schubert, E.: Nano Lett. 5(7), 1385 (2005)

69. Lee, Y., Ruby, D., Peters, D., McKenzie, B., Hsu, J.: Nano Lett. 8(5), 1501 (2008)

70. Chiu, M., Chang, C., Tsai, M., Chang, F., Yu, P.: Opt. Express 18(103), A308 (2010)

71. Chang, C., Hsu, M., Tseng, P., Yu, P., Chang, W., Sun, W., Hsu, W.: Opt. Express 19(103), A219 (2011)
72. Chang, C., Yu, P., Hsu, M., Tseng, P., Chang, W., Sun, W., Hsu, W., Hsu, S., Chang, Y.: Nanotechnology 22, 095201 (2011)

73. Yee, K.: IEEE Trans. Antennas Propag. 14(3), 302 (1966)

74. Taflove, A.: IEEE Trans. Electromagn. Compat. 3, 191 (1980)

75. Yu, W., Mittra, R., Yang, X., Liu, Y., Rao, Q., Muto, A.: IEEE Microw. Mag. 11(4), 43 (2010)

76. Krakiwsky, S., Turner, L., Okoniewski, M.: IEEE Microw. Symp. Dig. 2, 1033-1036 (2004)

77. Adams, S., Payne, J., Boppana, R.: hpcmp-ugc, pp. 334-338 (2007)

78. Sullivan, D.: IEEE Trans. Antennas Propag. 40(10), 1223 (1992)

79. Dou, L., Sebak, A.: Microw. Opt. Technol. Lett. 48(10), 2083 (2006)

80. Diedenhofen, S.L., Janssen, O.T.A., Grzela, G., Bakkers, E.P.A.M., Goómez Rivas, J.: ACS Nano 5(3), 2316 (2011)

81. Kelzenberg, M., Putnam, M., Turner-Evans, D., Lewis, N., Atwater, H.: In: IEEE Photovoltaic Specialists Conference, pp. 948 953 (2009)

82. Kelzenberg, M., Boettcher, S., Petykiewicz, J., Turner-Evans, D., Putnam, M., Waren, E., Spurgeon, E., Briggs, R., Lewis, N., Atwater, H.: Nat. Mater. 5(3), 948-953 (2010)

83. Yip, C.H., Chiang, Y.M., Wong, C.C.: J. Phys. Chem. C 112(23), 8735 (2008)

84. Yip, C., Chiang, Y., Wong, C.: J. Opt. Soc. Am. B 27(5), 920 (2010)

85. Li, J., Yu, H., Wong, S., Zhang, G., Sun, X., Lo, P., Kwong, D.: Appl. Phys. Lett. 95, 033102 (2009)

86. Kupec, J., Witzigmann, B.: Opt. Express 17(12), 10399 (2009)

87. Kupec, J., Stoop, R., Witzigmann, B.: Opt. Express 18(26), 27589 (2010)

88. Joannopoulos, J., Winn, J.: Photonic Crystals: Molding the Flow of Light. Princeton Univ. Press, Princeton (2008)

89. Pendry, J.: J. Mod. Opt. 41(2), 209 (1994)

90. Bell, P., Pendry, J., Moreno, L., Ward, A.: Comput. Phys. Commun. 85(2), 306 (1995)

91. Li, Z.Y., Lin, L.L.: Phys. Rev. E 67(4), 046607 (2003)

92. Whittaker, D.M., Culshaw, I.S.: Phys. Rev. B 60(4), 2610 (1999)

93. Hu, L., Chen, G.: Nano Lett. 7(11), 3249 (2007)

94. Lalanne, P., Silberstein, E.: Opt. Lett. 25(15), 1092 (2000)

95. Lalanne, P., Jurek, M.: J. Mod. Opt. 45(7), 1357 (1998)

96. Moharam, M., Gaylord, T.: J. Opt. Soc. Am. B 72(10), 1385 (1982)

97. Moharam, M., Pommet, D., Grann, E., Gaylord, T.: J. Opt. Soc. Am. A 12(5), 1077 (1995)

98. Moharam, M., Grann, E., Pommet, D., Gaylord, T.: J. Opt. Soc. Am. A 12(5), 1068 (1995)

99. Mallick, S., Agrawal, M., Peumans, P.: Opt. Express 18(6), 5691 (2010)

100. Popov, E., Nevière, M.: J. Opt. Soc. Am. A 18(11), 2886 (2001)

101. Li, L.: J. Opt. A, Pure Appl. Opt. 5, 345 (2003)

102. Anttu, N., Xu, H.: J. Nanosci. Nanotechnol. 10(11), 7183 (2010)

103. Matias, I., Del Villar, I., Arregui, F., Claus, R.: J. Opt. Soc. Am. A 20(4), 644 (2003)

104. Yang, F., Chen, J., Qiang, R., Elsherbeni, A.: In: IEEE Antennas and Propagation Soc. Int. Symposium, pp. 2715-2718 (2006)

105. Taflove, A., Hagness, S., et al.: Computational Electrodynamics: The Finite-Difference Time-Domain Method, vol. 347. Artech House, Boston (1995)

106. Harms, P., Mittra, R., Ko, W.: IEEE Trans. Antennas Propag. 42(9), 1317 (1994)

107. Lucas, E., Fontana, T.: IEEE Trans. Antennas Propag. 43(2), 145 (1995)

108. Vouvakis, M., Zhao, K., Lee, J.: IEEE Trans. Magn. 42(4), 691 (2006)

109. Duché, D., Escoubas, L., Simon, J., Torchio, P., Vervisch, W., Flory, F.: Appl. Phys. Lett. 92, 193310 (2008) 
110. Kupec, J., Akçakoca, U., Witzigmann, B.: J. Opt. Soc. Am. B 28(1), 69 (2011)

111. Ramahi, O., Mittra, R.: IEEE Trans. Magn. 25(4), 3043 (1989)

112. Eibert, T., Hansen, V.: J. Electromagn. Waves Appl. 10(1), 61 (1996)

113. Berenger, J.: J. Comput. Phys. 114(2), 185 (1994)

114. Gedney, S.: IEEE Trans. Antennas Propag. 44(12), 1630 (1996)

115. Pisarenco, M., Maubach, J., Setija, I., Mattheij, R.: J. Opt. Soc. Am. A 27(11), 2423 (2010)

116. Donges, A.: Eur. J. Phys. 19, 245 (1998)

117. Kupec, J., Yu, S., Witzigmann, B.: Proc. SPIE 7597, 759704 (2010)

118. Russer, P.: Electromagnetics, Microwave Circuit and Antenna Design for Communications Engineering. Artech House, Boston (2003)

119. Dong, Y., Tian, B., Kempa, T., Lieber, C.: Nano Lett. 9(5), 2183 (2009)

120. Borgström, M., Wallentin, J., Heurlin, M., Fält, S., Wickert, P., Leene, J., Magnusson, M., Deppert, K., Samuelson, L.: IEEE J. Sel. Top. Quantum Electron. 17(4), 1050 (2011)

121. Borgström, M., Norberg, E., Wickert, P., Nilsson, H., Trägårdh, J., Dick, K., Statkute, G., Ramvall, P., Deppert, K., Samuelson, L.: Nanotechnology 19, 445602 (2008)

122. Shockley, W., Queisser, H.: J. Appl. Phys. 32, 510 (1961)

123. Létay, G.: Modellierung von III-V Solarzellen. Doktorarbeit, Universität Konstanz, Deutschland (2003)

124. Castaner, L., Silvestre, S., Castaaner, L.: Modelling Photovoltaic Systems Using PSpice. Wiley Online Library (2002)

125. Yu, S., Kupec, J., Witzigmann, B.: IEEE NUSOD, 57-58 (2010)

126. Würfel, P.: Physics of Solar Cells. Wiley Online Library (2005)

127. Kayes, B., Atwater, H., Lewis, N.: J. Appl. Phys. 97, 114302 (2005)

128. Purcell, E., Torrey, H., Pound, R.: Phys. Rev. 69(1-2), 37 (1946)

129. Römer, F., Witzigmann, B., Chinellato, O., Arbenz, P.: Opt. Quantum Electron. 39(4), 341 (2007)

130. Tezuka, T., Nunoue, S., Yoshida, H., Noda, T.: Jpn. J. Appl. Phys. Lett. 32, 54 (1993)

131. David, A., Meier, C., Sharma, R., Diana, F., DenBaars, S., Hu, E., Nakamura, S., Weisbuch, C., Benisty, H.: Appl. Phys. Lett. 87, 101107 (2005)

132. David, A., Fujii, T., Moran, B., Nakamura, S., DenBaars, S., Weisbuch, C., Benisty, H.: Appl. Phys. Lett. 88, 133514 (2006)

133. Tripathy, S., Teo, S., Lin, V., Chen, M., Dadgar, A., Christen, J., Krost, A.: Phys. Status Solidi (c) 7(1), 88 (2010)

134. Fan, S., Villeneuve, P., Joannopoulos, J., Schubert, E.: Phys. Rev. Lett. 78(17), 3294 (1997)

135. David, A., Benisty, H., Weisbuch, C.: J. Disp. Technol. 3(2), 133 (2007)

136. Wiesmann, C., Bergenek, K., Linder, N., Schwarz, U.: Laser Photonics Rev. 3(3), 262 (2009)
137. Agarwal, G.: Phys. Rev. A 11(1), 253 (1975)

138. Römer, F., Witzigmann, B.: J. Opt. Soc. Am. B 25, 31 (2008)

139. Stefano, O., Fina, N., Savasta, S., Girlanda, R., Pieruccini, M.: J. Phys. 22, 315302 (2010)

140. Tsai, Y., Lin, C., Chang, J.: Opt. Rev. 16(3), 347 (2009)

141. Fussell, D., McPhedran, R., Martijn de Sterke, C.: Phys. Rev. E 70(6), 66608 (2004)

142. Stefano, O., Fina, N., Savasta, S., Girlanda, R., Pieruccini, M.: J. Phys. 22, 315302 (2010)

143. Rau, U.: Phys. Rev. B 76(8), 85303 (2007)

144. Catchpole, K.: Phil. Trans. R. Soc. A 364(1849), 3493 (2006)

145. Ries, H., Smestad, G., Winston, R.: Proc. SPIE 1528, 7 (1991)

146. Létay, G., Bett, A.: Spectrum 20, 25 (2001)

147. Smestad, G., Ries, H., Winston, R., Yablonovitch, E.: Sol. Energy Mater. 21(2-3), 99 (1990)

148. Derkacs, D., Lim, S., Matheu, P., Mar, W., Yu, E.: Appl. Phys. Lett. 89, 093103 (2006)

149. Schaadt, D., Feng, B., Yu, E.: Appl. Phys. Lett. 86, 063106 (2005)

150. Catchpole, K., Polman, A.: Opt. Express 16(26), 21793 (2008)

151. Steiger, S., Veprek, R., Witzigmann, B., Comp, J.: Electronics 7(4), 509 (2008)

152. Steiger, S., Veprek, R., Witzigmann, B.: Opt. Quantum Electr. 1-7 (2009)

153. Ferry, V., Verschuuren, M., Li, H., Verhagen, E., Walters, R., Schropp, R., Atwater, H., Polman, A.: Opt. Express 18 (2010)

154. Miñano, J., Luque, A., Tobías, I.: Appl. Opt. 31(16), 3114 (1992)

155. Fahr, S., Ulbrich, C., Kirchartz, T., Rau, U., Rockstuhl, C., Lederer, F.: Opt. Express 16(13), 9332 (2008)

156. Ulbrich, C., Peters, M., Bläsi, B., Kirchartz, T., Gerber, A., Rau, U.: Opt. Express 18(S2), A133 (2010)

157. Peters, M., Goldschmidt, J., Kirchartz, T., Bläsi, B.: Sol. Energy Mater. Sol. Cells 93(10), 1721 (2009)

158. Peters, M., Ulbrich, C., Goldschmidt, J., Fernandez, J., Siefer, G., Bläsi, B.: Opt. Express 19(102), A136 (2011)

159. Goetzberger, A., Goldschmidt, J., Peters, M., Löper, P.: Sol. Energy Mater. Sol. Cells 92(12), 1570 (2008)

160. Peters, M., Goldschmidt, J., Bläsi, B.: Sol. Energy Mater. Sol. Cells 94(8), 1393 (2010)

161. Ulbrich, C., Fahr, S., Üpping, J., Peters, M., Kirchartz, T., Rockstuhl, C., Wehrspohn, R., Gombert, A., Lederer, F., Rau, U.: Phys. Status Solidi (a) 205(12), 2831 (2008)

162. Adams, J., Elder, W., Stavrinou, P., Roberts, J., Gonzalez, M., Tischler, J., Walters, R., Abell, J., Vurgaftman, I., Meyer, J., et al.: In: IEEE Photovoltaic Specialists Conference, pp. 1-5 (2010)

163. Adams, J., Browne, B., Ballard, I., Connolly, J., Chan, N., Ioannides, A., Elder, W., Stavrinou, P., Barnham, K., Ekins-Daukes, N.: Prog. Photovoltaics Res. Appl. 19(7), 865 (2011)

164. Yang, P., Yan, R., Fardy, M.: Nano Lett. 10(5), 1529 (2010)

165. Nozik, A.J.: Nano Lett. 10(8), 2735 (2010) 